\title{
Interventional Study Protocol Version Blinded Role Code
}

National Cancer Institute

\section{Source}

National Cancer Institute. Interventional Study Protocol Version Blinded Role Code. NCI

Thesaurus. Code C93852.

A coded value specifying the roles of individuals who are masked for single or double blind studies. 